\title{
On the film density using high power impulse magnetron sputtering
}

\author{
Mattias Samuelsson, Daniel Lundin, Jens Jensen, Michael A Raadu, \\ Jon Tomas Gudmundsson and Ulf Helmersson
}

\section{Linköping University Post Print}

N.B.: When citing this work, cite the original article.

Original Publication:

Mattias Samuelsson, Daniel Lundin, Jens Jensen, Michael A Raadu, Jon Tomas Gudmundsson and Ulf Helmersson, On the film density using high power impulse magnetron sputtering, 2010, SURFACE and COATINGS TECHNOLOGY, (205), 2, 591-596.

http://dx.doi.org/10.1016/j.surfcoat.2010.07.041

Copyright: Elsevier http://www.elsevier.com/

Postprint available at: Linköping University Electronic Press http://urn.kb.se/resolve?urn=urn:nbn:se:liu:diva-60690 


\title{
On the film density using high power impulse magnetron sputtering
}

\author{
Mattias Samuelsson ${ }^{1,2, *}$, Daniel Lundin ${ }^{1}$, Jens Jensen ${ }^{3}$, Michael A. Raadu ${ }^{4}$, Jon Tomas \\ Gudmundsson $^{5,6}$ and Ulf Helmersson ${ }^{1}$
}

${ }^{1}$ Plasma \& Coatings Physics Division, IFM-Materials Physics, Linköping University, SE-581 83 Linköping, Sweden

${ }^{2}$ Impact Coatings, Westmansgatan 29, SE-582 16 Linköping, Sweden

3 Thin Film Physics Division, IFM-Materials Physics, Linköping University, SE-581 83 Linköping, Sweden

${ }^{4}$ Division of Space and Plasma Physics, School of Electrical Engineering, Royal Institute of Technology, SE-100 44 Stockholm, Sweden

${ }^{5}$ Science Institute, University of Iceland, Dunhaga 3, IS-107 Reykjavik, Iceland

${ }^{6}$ Department of Electrical and Computer Engineering, University of Iceland, Hjardarhaga 26, IS-107Reykjavik, Iceland

* Corresponding author. E-mail: matsa757@ifm.liu.se

Complete Address: Mattias Samuelsson

Department of Physics, Chemistry \& Biology,

Linköping University,

SE-581 83, Linköping,

Sweden.

Office Phone: $\quad$ 0046-13-282712

Fax: $\quad$ 0046-13-137568

\begin{abstract}
The influence on thin film density using high power impulse magnetron sputtering (HiPIMS) has been investigated for eight different target materials ( $\mathrm{Al}, \mathrm{Ti}, \mathrm{Cr}, \mathrm{Cu}, \mathrm{Zr}, \mathrm{Ag}, \mathrm{Ta}$, and $\mathrm{Pt}$ ). The density values as well as deposition rates have been compared to results obtained from thin films grown by direct current magnetron sputtering (DCMS) under the same experimental conditions. Overall, it was found that the HiPIMS deposited coatings were approximately 5-15\% denser compared to the DCMS deposited coatings. This could be attributed to the increased metal ion bombardment commonly seen in HiPIMS discharges, which also was verified using a global plasma model to assess the degree of ionization of
\end{abstract}


sputtered metal. One key feature is that the momentum transfer between the growing film and the incoming metal ions is very efficient due to the equal mass of film and bombarding species, leading to a less pronounced columnar microstructure. As expected the deposition rates were found to be lower for HiPIMS compared to DCMS. For several materials this decrease is not as pronounced as previously reported in the literature, which is shown in the case of $\mathrm{Ta}, \mathrm{Pt}$, and $\mathrm{Ag}$ with rate $_{\mathrm{HiPIMS}} /$ rate $_{\mathrm{DCMS}} \sim 70-85 \%$, while still achieving denser coatings.

Keywords: HiPIMS; HPPMS; DCMS; Density; RBS; Global Plasma Model 


\section{Introduction}

High power impulse magnetron sputtering (HiPIMS) is a promising technique for improving magnetron sputtering today being used in many industrial processes for thin film deposition [1]. The HiPIMS discharge generates large quantities of highly energetic ions [2] due to very high pulse power densities with, in some cases, a directed flux of charged species [3]. The transport of these energetic particles is not fully understood, but it is clear that they have a dramatic effect on thin film growth, such as densification and improved adhesion [4].

Previous studies on HIPIMS have shown that for some cases the increase in thin film density can be as much as $30 \%$ [5], whereas other reports claim slightly more than $10 \%$ [6] compared with conventional techniques such as direct current magnetron sputtering (DCMS) or mid-frequency asymmetric bipolar pulsed magnetron sputtering. In a recent study on $\mathrm{CrN}$ deposition, Alami et al. measure a density increase of about $6 \%$ when depositing with HiPIMS compared to DCMS, although the stoichiometry of the deposited films was not reported [7]. These values depend not only on process conditions and system configuration, but also heavily on source material, making it difficult to draw any conclusions regarding trends in film densification between separate studies. This stresses the need for the present study of single element metal sputtering processes, keeping the complexity and variation in process conditions at a minimum. By doing so, it may be possible to determine whether or not there is an overall trend in change of film density when using HiPIMS as compared to DCMS. It is of essence to investigate this property, since the film density is an important parameter for many types of coatings, such as mass-diffusion barriers, thermal barriers as well as for corrosion protection and wear resistance.

There are indications in the literature on film densification mechanisms that may give valid information to explain the observed behaviour for the HiPIMS films density increase. From transmission electron microscopy studies by Petrov et al. [8] it is known that an increase in the ion flux greatly reduces intracolumnar as well as intercolumnar porosity, 
where it is suggested that the incoming ions collisionally enhance the surface mobility. Molecular dynamics simulations of multilayer thin film growth when varying the ion/metal flux ratio also show that the film roughness is reduced for an increased ion flux [9]. This has also been experimentally confirmed by Eriksson et al. [10], and they conclude that ion assistance leading to a higher ion/metal flux ratio results in an increased ad-atom mobility and a smoothening effect. They also point out that for ion energies in the range $20-30 \mathrm{eV}$ the coatings become denser. In a dedicated HiPIMS study by Alami et al. [4], the importance of a highly metallic ion deposition flux for film densification is also stressed. From ion energy measurements in HiPIMS discharges it has been found that the average metal ion energy is around $20 \mathrm{eV}$, whereas the argon gas ions show much lower average energies $(<10 \mathrm{eV}$ or less), without using any substrate bias [2,3].

Furthermore, a high ionized flux fraction has been demonstrated in several independent works on HiPIMS. The fraction of ionized metal has been shown to reach above $90 \%$ for $\mathrm{Ti}[11]$. For $\mathrm{Cu}$ the measured ionized flux fraction was estimated to approximately $70 \%$ by Kouznetsov et al. [12] and $\mathrm{Cu}^{+}$was measured to be almost $92 \%$ of the total ion flux to the substrate as reported by Vlcek et al. [13]. For conventional magnetron sputtering discharges, the ionized flux fraction is of the order of a few percent [14]. It is therefore suggested that the discharge conditions for HiPIMS are such that both a high relative flux of metal ions as well as the desired ion energy are present.

Also reported for HiPIMS processes is the lower deposition rate as compared to DCMS values $[1,15,16]$ under similar conditions. There are likely a number of reasons for this behaviour such as back-attraction of ionized sputtered material [15], the magnetic arrangement and anomalous transport of charged species in HiPIMS discharges, which is more thoroughly discussed in a previous publication [3]. In general, it is clear that the transport of the sputtered material is greatly affected by electric and magnetic fields when a large fraction is ionized as in the case of HiPIMS. This can also be seen when comparing the 
deposition rates for different magnetic field configurations (see for example references 17 and 18). Konstantinidis et al. also show that the relative deposition rate in the case of Ti changes dramatically from $20 \%$ to $70 \%$ when decreasing the pulse length [19] and thereby affecting the discharge characteristics such as level of self sputtering taking place as well as neutral gas heating followed by gas depletion and change of plasma conductivity [20]. Furthermore, in a work by Helmersson et al. [21] it was shown that the reduction in relative deposition rate was particularly pronounced for metals with a low self-sputtering yield.

In this study eight different metallic materials ( $\mathrm{Al}, \mathrm{Ti}, \mathrm{Cr}, \mathrm{Cu}, \mathrm{Zr}, \mathrm{Ag}, \mathrm{Ta}$, and $\mathrm{Pt}$ ), all relevant for many deposition processes, have been chosen for a more detailed study of the film density and deposition rates when using HiPIMS. No compound materials were used in order to minimize the complexity of the system. The density values were measured and compared with results from thin films grown under equivalent conditions using DCMS in addition to tabulated bulk values. The impurity content and its effect on the density have also been analyzed. The overall results were compared to a global plasma model providing insights on the ionization of the sputtered metal ions, which subsequently are transported to the substrate during growth.

\section{Methods and materials}

\subsection{Experimental details}

The thin films investigated in this work were deposited in a high vacuum deposition system. Here, a planar circular $\Phi=0.076 \mathrm{~m}$ magnetron (Gencoa Ltd) equipped with different commercially available metal targets (Al, Ti, Cr, Cu, Zr, Ag, Ta, and Pt), having a thickness of $0.003175 \mathrm{~m}(1 / 8 \mathrm{inch})$, with the exception of Ta, being $0.00635 \mathrm{~m}(1 / 4$ inch $)$ thick. The target purities varied depending on material. Since commercial targets were used, the purity was in all cases above 99\%, which was verified by the RBS and ERDA analyses. The magnetron was mounted in a cylindrical vacuum chamber (height $0.30 \mathrm{~m}$, diameter $0.42 \mathrm{~m}$ ) pumped with a turbo-molecular pump to a background pressure of about $7 \times 10^{-5} \mathrm{~Pa}$, after 
which Ar, with a minimum purity of $99.9997 \%$, was leaked into the chamber. The discharge pressure was $0.67 \mathrm{~Pa}$ unless otherwise stated. Si substrates were placed on a static substrate holder at a distance of $0.065 \mathrm{~m}$ from the target surface. The substrate holder was grounded. All depositions were performed at ambient temperature, and at least four samples were deposited for each process condition. In this investigation the average power was kept constant at $125 \mathrm{~W}$ for both deposition methods, except in the case of Pt and Ta where the average power was slightly lower due to voltage limitations of the charging power supply. In the case of HiPIMS, unipolar voltage pulses were applied to the magnetron by a pulsing unit (SPIK 1000A, Melec GmbH) charged by a direct current (DC) power supply (Pinnacle, Advanced Energy) delivering constant voltage pulses of controlled length. The specific voltage and current characteristics varied depending on target materials, but typical values of $\sim 880 \mathrm{~V}$ and $\sim 30 \mathrm{~A}$ were used generating approximately $100 \mu \mathrm{s}$ discharge pulses at $100 \mathrm{~Hz}$. Since the power pulse characteristics were fed into the global plasma model described below, it is here worth mentioning that little difference in the appearance of the resulting power pulse characteristics between the different target materials was seen.

For DCMS, a DC power supply (MDX 1K, Advanced Energy) was connected to the magnetron set to deliver a constant power of $125 \mathrm{~W}$.

The density $(\rho)$ of the deposited thin films was obtained by measuring the areal atomic density $\left(N_{S}\right)$ by Rutherford backscattering spectrometry (RBS) and the film thickness $(t)$ independently. For a single element film the density is then calculated as $\rho=\left(N_{S} / t\right) \times\left(M / N_{A}\right)$ [22], where $M$ is the atomic mass and $N_{\mathrm{A}}$ is Avogadro's number. This method has previously been used by Wang et al. to successfully evaluate thin film densities [23]. In order to measure the film thickness a scanning electron microscope (SEM, LEO 1550 Gemini) as well as a stylus profilometer (Vecco Dektak 6M) were employed. Both the aforementioned methods were used on all films in determining the film thickness mean value. The accuracy of the SEM were in this work a few tens of $\mathrm{nm}$ and the thickness values were averaged over a $1 \mathrm{~cm}$ 
wide cross section of the film in order to minimize any errors. In the case of the profilometer it has a maximum vertical resolution of $10 \mathrm{~nm}$. To facilitate reliable RBS analysis, all film thicknesses were between 350 and $500 \mathrm{~nm}$.

The RBS measurements were performed at the tandem accelerator of Uppsala University, using a $2.0 \mathrm{MeV}{ }^{4} \mathrm{He}^{+}$beam with a backscattering angle of $172^{\circ}$. The incident angle was $6^{\circ}$ with respect to the surface normal (to avoid channelling effects in the substrate).

The obtained spectra were evaluated using the SIMNRA 6.04 code [24]. For RBS, the dominant contribution to the uncertainty is associated with the uncertainty in stopping cross section for the respective elements [22]. The error is typically $3-5 \%$, and in the present study $5 \%$ was chosen for all elements. In order not to underestimate the error for the density calculations, a minimum and maximum value for the density was calculated using the standard error for the RBS results and the extreme values in the film thickness determination. The error bars for the density values thus serves as an upper limit of the actual error. Furthermore, time-of-flight elastic recoil detection analysis (ToF-ERDA) [25] was employed to estimate the level of impurities hidden in the RBS spectra. As primary projectile $40 \mathrm{MeV}$ ${ }^{127} \mathrm{I}^{9+}$ ions were used, the incident angle was $22.5^{\circ}$ and the detector was placed at a recoil scattering angle of $45^{\circ}$. The information gained from the ToF-ERDA measurements was fed back to the SIMNRA evaluation in order to better reflect the composition of the measured samples.

\subsection{Plasma modelling}

In this work, a new, time-dependent (zero-dimensional) plasma model based on works by Ashida et al. [26], Hopwood [27], and Gudmundsson [28] was employed to simulate ionization and gas dynamics during the HiPIMS discharge. The model operates by solving the coupled differential equations, essentially similar to those given in detail by Gudmundsson [28]. However, in the present model the production and loss of ions, electrons, and neutral species is assumed to take place in an annular region above the target race track, $0.04 \mathrm{~m}$ wide 
(inner radius $0.025 \mathrm{~m}$ and outer radius $0.065 \mathrm{~m}$ ), and extending outwards from the target surface defined by $z_{1}<z<z_{2}$, where $z_{1}=0.00 \mathrm{~m}$ and $z_{2}=0.02 \mathrm{~m}$. This volume constitutes our ionization region, where we expect the ionization to be most pronounced. Geometrical effects are included indirectly when calculating diffusion losses and input of sputtered material, although the treatment is essentially zero-dimensional, i.e. only volume average values are calculated. Besides the smaller volume used in the present calculations, it also includes new effects such as the enhanced depletion of neutral atoms by collisions with fast metal ions as well as a balance with metastable Ar atoms, and charge exchange between Ar ions and metal atoms, and de-excitation of metastable Ar atoms due to Penning ionization of metal atoms. This means that the model can also be used to investigate time dependent effects of neutral gas depletion, which will be explored in a future work. A detailed analysis of the new equations and their effects on the neutral kinetics is beyond the scope of the present investigation, since the aim has been to model the ionization of sputtered metal.

To set up equations for the rate of change of the different species in the global model it is necessary to know the reaction rates, $k_{\text {reaction }}$. These were taken from Hopwood [27], Lee $e t$ al. $[29,30]$, and calculated from the electron impact ionization cross sections given by Freund et al. [31]. During the discharge pulse electron impact ionization is the most effective process in creating metal ions $\left(e+M \rightarrow M^{+}+2 e\right)$ in the HiPIMS discharge [28] and the rate coefficients $\left(k_{m i z}\right)$ for such an event are listed in Table 1. Also given is the ionization mean free path for the sputtered metal neutral calculated during the current peak of the pulse $(t \sim 55$ $\mu \mathrm{s})$, expressed as $\lambda_{\text {miz }}=v_{s} / k_{\text {miz }} n_{e}$. Here, $v_{s}$ is the velocity of the sputtered metal neutral and is based on measurements by Britun et al. [32]. Other basic plasma parameters needed, such as the electron density, $n_{e}$, and the electron temperature, $T_{e}$, have been extracted from the global model for $t \sim 55 \mu \mathrm{s}$. By using the above information a simple example of how the density of metal ions changes in time can thereby be modeled as 


$$
\frac{d n_{M^{+}}}{d t}=k_{\text {miz }} n_{e} n_{M}+k_{\text {chexc }} n_{A r^{+}} n_{M}-k_{\text {wall }, M^{+}} n_{M^{+}},
$$

where the rates for electron impact ionization, charge exchange and loss of ions to chamber walls describe the different processes taking place. After solving this set of balance equations the degree of ionization for sputtered metal can be calculated as $\left(n_{M^{+}}\right) /\left(n_{M}+n_{M^{+}}\right)$, where M is the metal neutral and $\mathrm{M}^{+}$the singly charged metal ion. No multiply charged ions were taken into account in these calculations.

The model uses an experimentally determined time profile for the electrical power input [28]. The fraction of the power assumed to be deposited in the ionization region is determined by matching the measured current to the calculated total ion current to the target. This was done in order to benchmark the calculated results to what was measured. From a plasma point of view this means that in the model it is not assumed that all the applied power is directly delivered to the ionization region. The fact that we have a large potential drop over the cathode sheath, accelerating ions to the target, means that a large fraction of the power is lost to the sheath region, which is now accounted for. In these calculations, a typical HiPIMS pulse used in the experiments was chosen to approximate the pulse for all target materials. The discharge voltage was therefore set to $880 \mathrm{~V}$, the current peaked at $30 \mathrm{~A}$, and the pulse length used was $100 \mu \mathrm{s}$.

\section{Results and discussion}

\subsection{Deposition rate}

The deposition rates for the different target materials used as well as the relative deposition rates (rate HiPIMS $_{\text {rate }}$ DCMS) are plotted in Figure 1 for both DCMS and HiPIMS using the same average power for each target comparison. As an example, in this work the HiPIMS deposition rate of Ti was found to be approximately $30 \%$ of the DCMS rate. Using roughly the same discharge voltage and current on another magnetron this fraction was determined to about $21 \%$ [3]. The previously discussed dependence of relative deposition rate on self- 
sputter yield as reported by Helmersson et al. [21] could not be observed for the current experimental setup. As shown in Figure $1 \mathrm{Cr}, \mathrm{Zr}$ and $\mathrm{Al}$ have almost the same relative deposition rate (47\%-49\%), even though $\mathrm{Cr}$ has a self-sputtering yield of 1.49 compared to 0.74 for $\mathrm{Zr}$ and 1.23 for $\mathrm{Al}$, as simulated by SRIM-2008 using an incoming energy of $700 \mathrm{eV}$ [33]. In all cases the applied peak voltage did not vary much from the value used in the SRIM simulations, which means that the effect of the difference in energy of the incoming sputtering metal ion can be neglected.

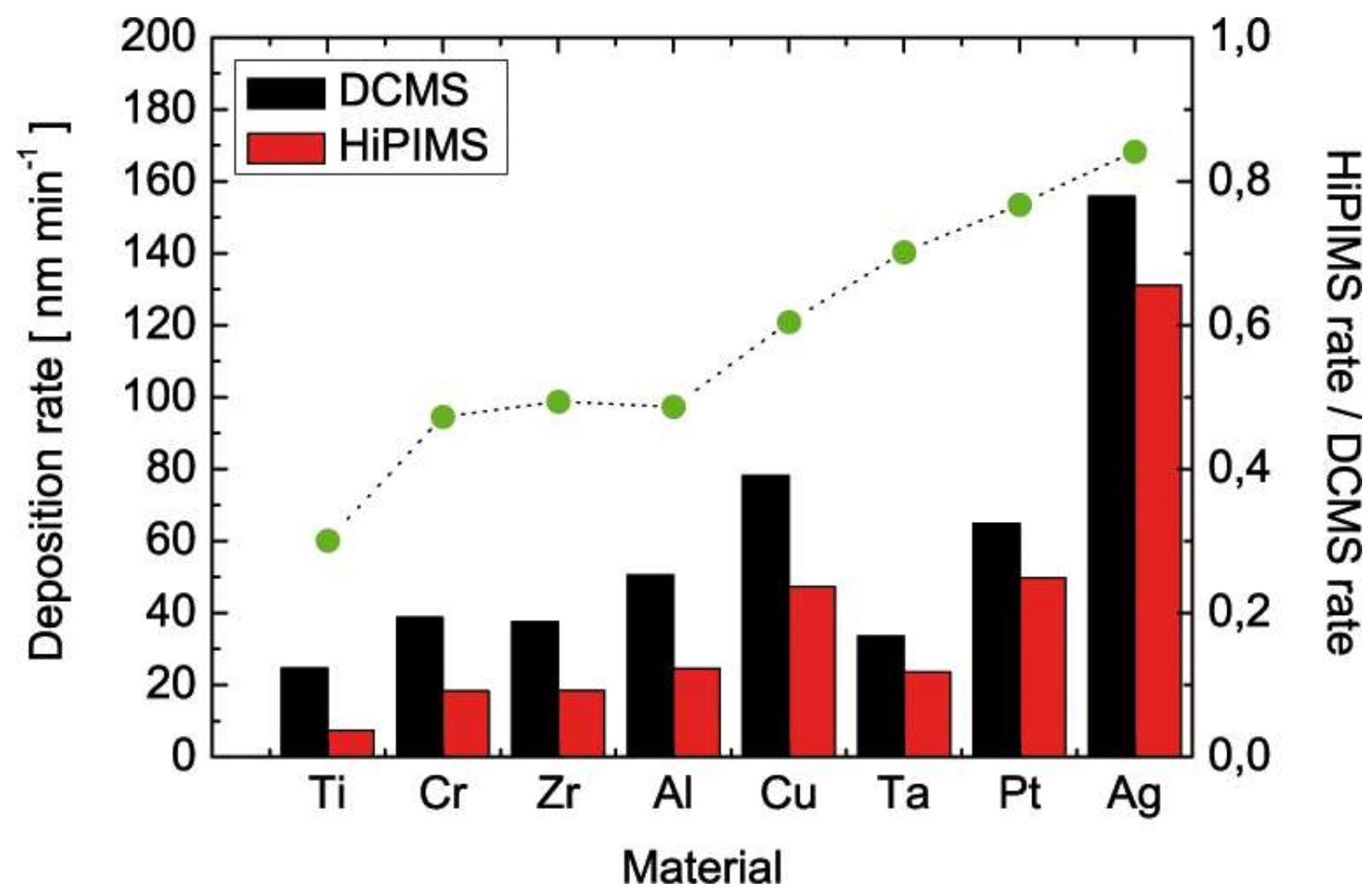

Figure 1. The deposition rates for DCMS and HiPIMS discharges plotted as bars for the different target materials used (left axis). The deposition rate of HiPIMS over DCMS deposition rate is shown as a scatter plot (right axis).

A comparison of the measured relative deposition rates between the results presented here and those of Helmersson et al. [21] is displayed in Figure 2, which emphasizes the fact that the reduction of deposition rate varies dramatically between different experimental setups as described in the introduction, and it is very likely that there is no single factor that can explain this difference. For the present experimental setup, a generally higher relative 
deposition rate is observed as compared to the values reported in reference 21 , reaching about $70-85 \%$ for Ta, Pt and Ag.

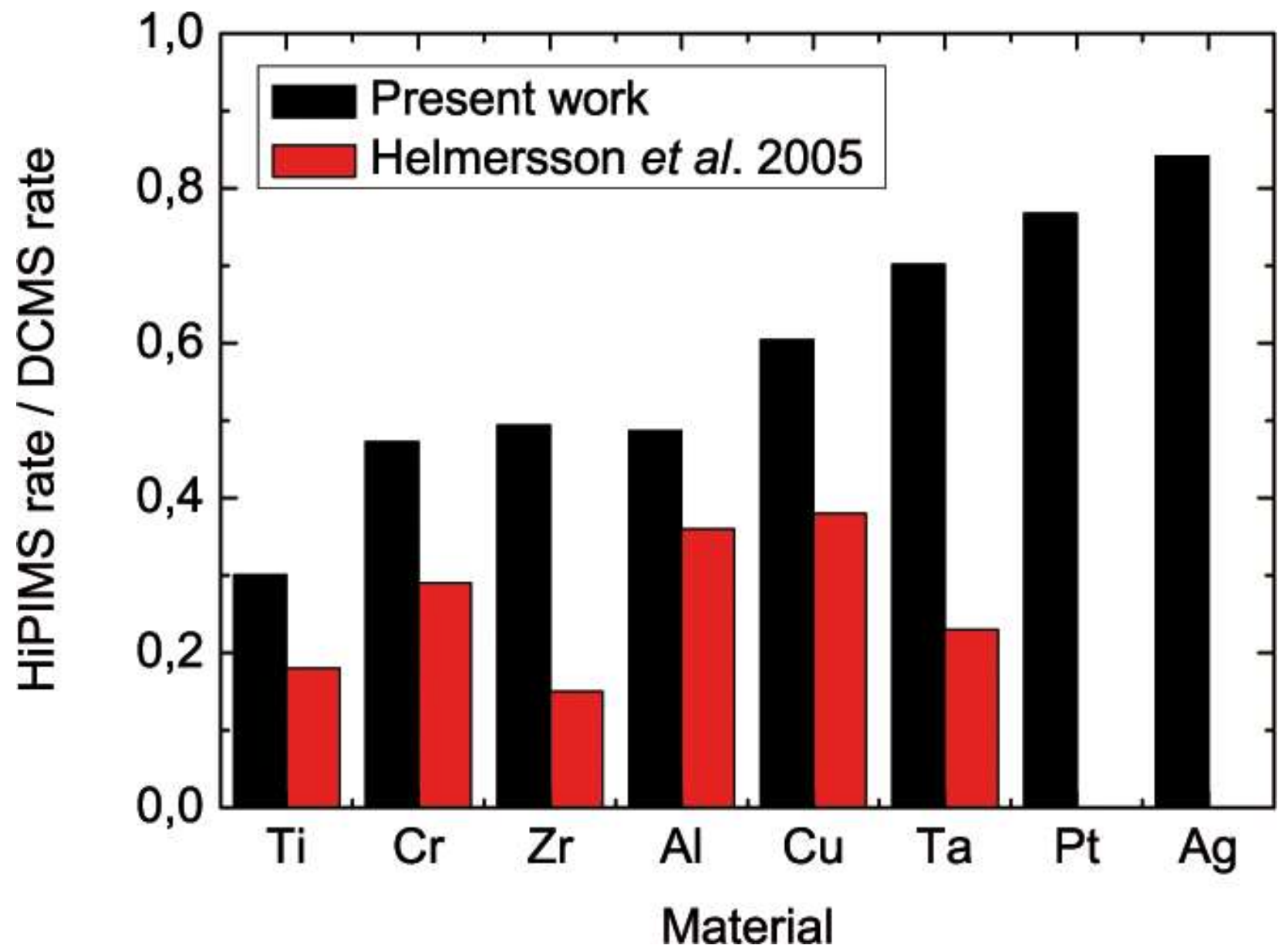

Figure 2. The measured relative deposition rate for the eight different target materials investigated in this work compared with previous results from Helmersson et al. [21]. Note that $\mathrm{Pt}$ and $\mathrm{Ag}$ was not included in the latter study.

\subsection{Film density}

The measured film density for films deposited from the different target materials on $\mathrm{Si}$ substrates is plotted in Figure 3. The values are estimated for films grown by DCMS and HiPIMS using RBS, SEM, and stylus profilometry. The results are compared to the bulk density given in literature [34]. In general a small increase in thin film density on the order of $5-15 \%$ can be seen when changing from DCMS to HiPIMS, with the exception of $\mathrm{Cu}$ and $\mathrm{Zr}$, showing near equal density values for both techniques. This cannot be explained by looking at the metal self-sputtering yield, since $\mathrm{Cu}$ has a very high yield, whereas $\mathrm{Zr}$ exhibits a low self-sputtering yield. Furthermore, ToF-ERDA analysis of selected samples shows very 
low concentration of contaminations (sub-percent levels) in the bulk of the film. One investigated target material $(\mathrm{Ta})$ had to be deposited at a higher process pressure $(1.33 \mathrm{~Pa})$, since the target thickness was too great, leading to insufficient magnetic confinement for a stable HiPIMS discharge to be maintained at lower pressures. As a consequence of this higher pressure, the Ta films show a presence of $2-3.5 \%$ Ar. All detected contaminations were taken into consideration in the SIMNRA simulations. It is therefore suggested that any detected deviation from tabulated bulk densities stem from a decrease of film density due to e.g. a porous microstructure.

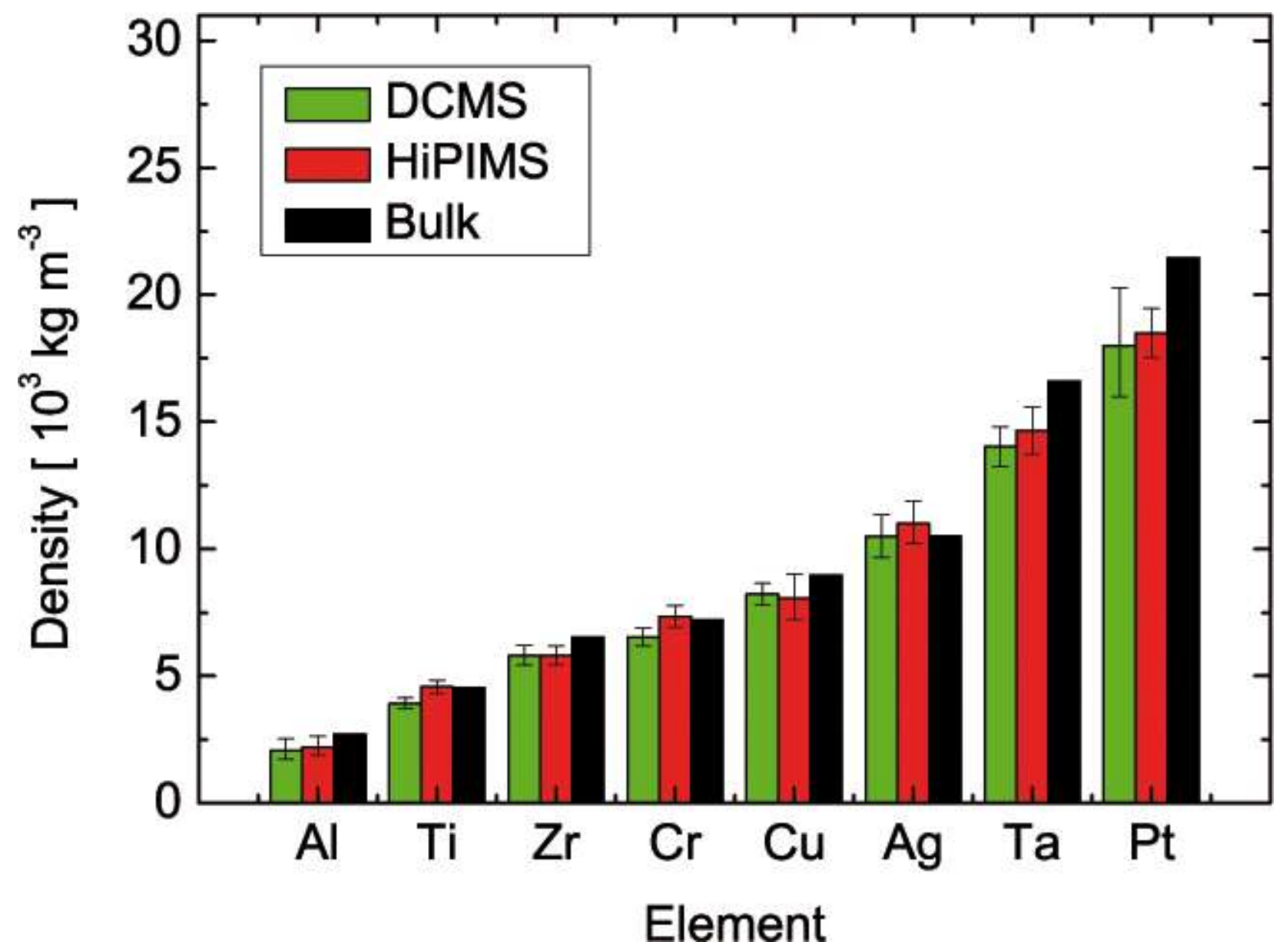

Figure 3. Thin film density plot for different target materials. The values are calculated for films grown by DCMS and HiPIMS using RBS, SEM and profilometry. The results are compared to the bulk density given in literature.

Figure 4 shows cross-sectional SEM images of the microstructure of two Ti samples grown by DCMS and HiPIMS respectively. In this figure the previously measured density difference ( $15 \%$ for Ti) can be perceived by simply comparing the two SEM viewgraphs, where the coating grown by HiPIMS clearly exhibits a denser microstructure. Although the 
comparison between film density as obtained by RBS and SEM is in agreement for Ti in the present case, it is difficult to draw any conclusions regarding density based solely upon information obtained from SEM images. This because films appearing fully dense can contain micro voids only visible in transmission electron microscopy studies. It should be noted that although density difference may be present (as indicated in Figure 3), this need not be visible in SEM.

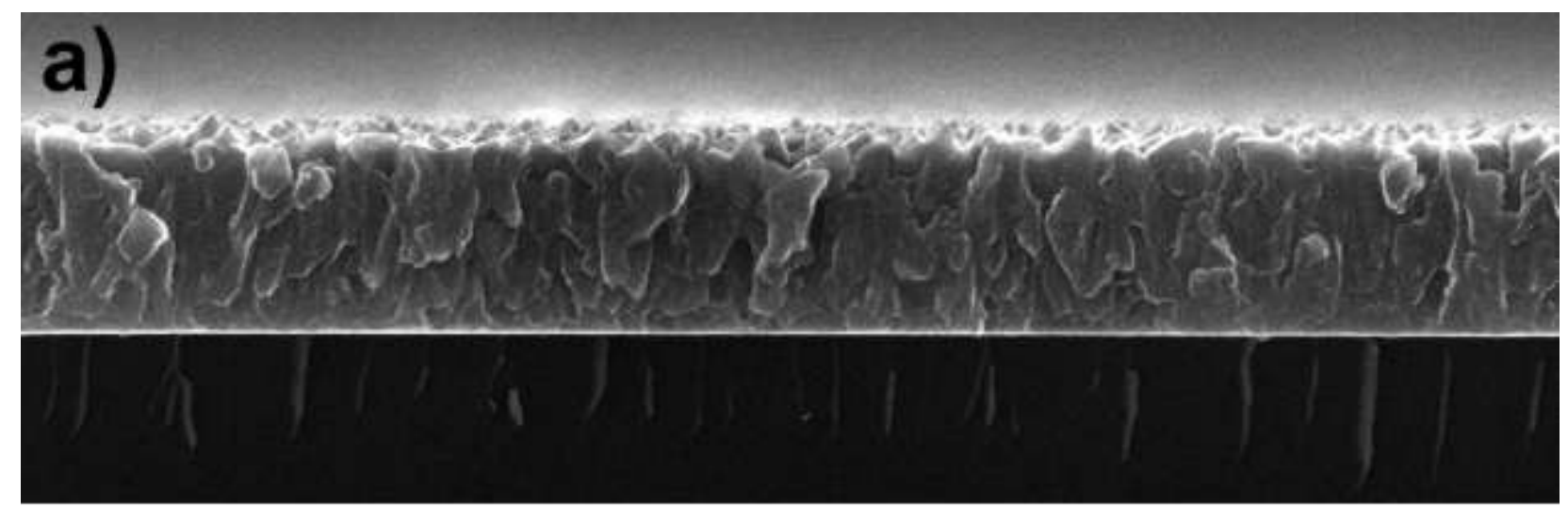

\section{b)}

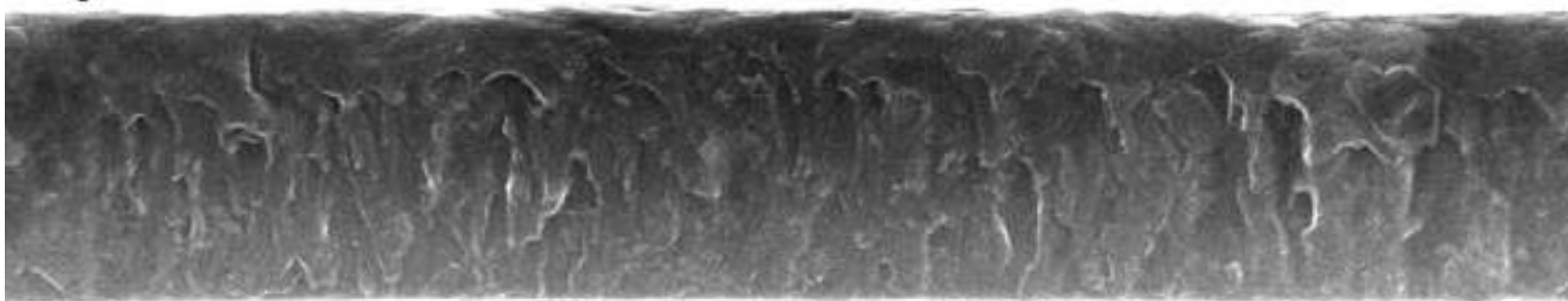

$400 \mathrm{~nm}$

Figure 4. Cross-sectional SEM image of a Ti sample grown by a) DCMS and b) HiPIMS. The DCMS deposited sample exhibits a porous microstructure and rough surface, whereas the HiPIMS deposited sample exhibits a less pronounced columnar microstructure and a smooth surface. The scale bar applies to both images.

In a few cases, such as for $\mathrm{Ag}$ and $\mathrm{Cr}$, the measured film density is slightly higher than the reported bulk value. So far, no explanation has been found, since the samples show very low levels of impurities and two separate methods have been used to evaluate the film thickness. On the other hand, the reported bulk values are within the limits of the achieved accuracy for the density values obtained in this study. 
Owing to the fact that the density value errors are overestimations and consequently there is an overlap of error bars, it is difficult to conclude from Figure 3 if there is a valid trend in the density increase seen when using HiPIMS. A significance test has been performed on the results in order to establish if there is significant general trend in density difference between the two deposition methods. In the test procedure the eight different targets are treated as independent measurements and the error estimations are assumed to have a normal distribution, where the extreme values fall within two standard deviations of the mean value, i.e. the values spanning the error bars in Figure 3 constitute $95 \%$ of all possible outcomes (see e.g. [35]). Furthermore, we need to introduce

$$
Z_{i}=\operatorname{HiPIMS}_{i}-\mathrm{DCMS}_{i} \square N\left(\mu_{Z_{i}}, \sigma_{i}^{2}\right),
$$

where $\mathrm{Z}_{\mathrm{i}}$ is a normal distribution based on the two normally distributed measurements from the experiment for target material $i . \mu$ and $\sigma^{2}$ are respectively the mean value and the variance. It is then easy to sum up the results for the eight different targets, and one arrives at

$$
\bar{Z}=\frac{1}{n} \sum_{j=1}^{n} Z_{j} \square N\left(\mu_{\bar{Z}}, \sigma_{t o t}^{2}\right),
$$

from which $\bar{Z}$ is calculated as the averaged observed difference in density from all the measurements, where $\sigma_{\text {tot }}^{2}=\frac{1}{n^{2}} \sum_{j=1}^{n} \sigma_{j}^{2}$, and we arrive at the following formulation of the problem

$$
\mu_{\bar{Z}}>\left[\bar{Z}-\sigma_{t o t} \Phi(1-\alpha)\right]>0
$$

where $\alpha$ is the significance level. Inserting the results from the measurements we arrive at $\Phi(1-\alpha)<1.83$, from which it can be concluded that our significance level is 0.035 , or in other words that there is a $96.5 \%$ probability that the HiPIMS coatings are denser than the DCMS coatings in the present study.

Here, it is proposed that the main mechanism for densification seen in HiPIMS is the energetic bombardment of the growing film structure. Here, several factors are here important, 
but the most crucial ones are the incident ion energy as well as the ionized metal flux fraction, $\Gamma_{M^{+}} /\left(\Gamma_{M^{+}}+\Gamma_{M}\right)$, where $\Gamma_{M^{+}}$is the metal ion flux to the substrate and $\Gamma_{M}$ the metal neutral flux. It should be stressed that the flux of sputter gas, such as Ar in this case, also should be taken into account in the overall process, but is here omitted for means of simplification. The simulated values for ion flux obtained from the model are thus underestimations of actual values since gas ions and neutral particles partake in the bombardment, and thus contribute to the energy transfer of the growing films. This limitation in the assumptions is not too severe considering the fact that the ion flux to the substrate is reported to be dominated by metal ions in HiPIMS processes [13].

\subsection{Plasma model}

The global time-dependent plasma model was employed to estimate the degree of ionization of the sputtered metal, $\left(n_{M^{+}}\right) /\left(n_{M}+n_{M^{+}}\right)$, depending on electron density and target material for a typical HiPIMS discharge.. These results are presented in Figure 5, which shows the metal ionization fraction for the sputtered vapour for the various metal targets. The ionization fraction for $\mathrm{Ti}$ reaches $56 \%$ roughly $55 \mu$ s into the pulse, while $\mathrm{Cu}$ reaches an ionization fraction of $27 \%$. It can be seen that, although $\mathrm{Ti}$ has a higher ionization potential than $\mathrm{Al}$, the ionization fraction for $\mathrm{Ti}$ is higher (56\% at the peak) than for $\mathrm{Al}(49 \%$ at the peak), since the electron impact ionization collision cross section is twice as high as that for $\mathrm{Al}$ and the mobility of $\mathrm{Ti}^{+}$being slightly lower. $\mathrm{Cu}$ on the other hand has higher ionization threshold than $\mathrm{Ti}$ or $\mathrm{Al}$ and a much lower electron impact ionization collision cross section, which explains the lower ionization fraction. Carbon, although not included in the experimental part of the present work, is added to the simulation results as a benchmark to the simulations. The model was also used to estimate values of the degree of ionization for DCMS under equivalent process conditions. It was found that in all cases it reached typically $5 \%$ or less, which was also reported by Rossnagel et al. [14] and Hopwood [27]. 


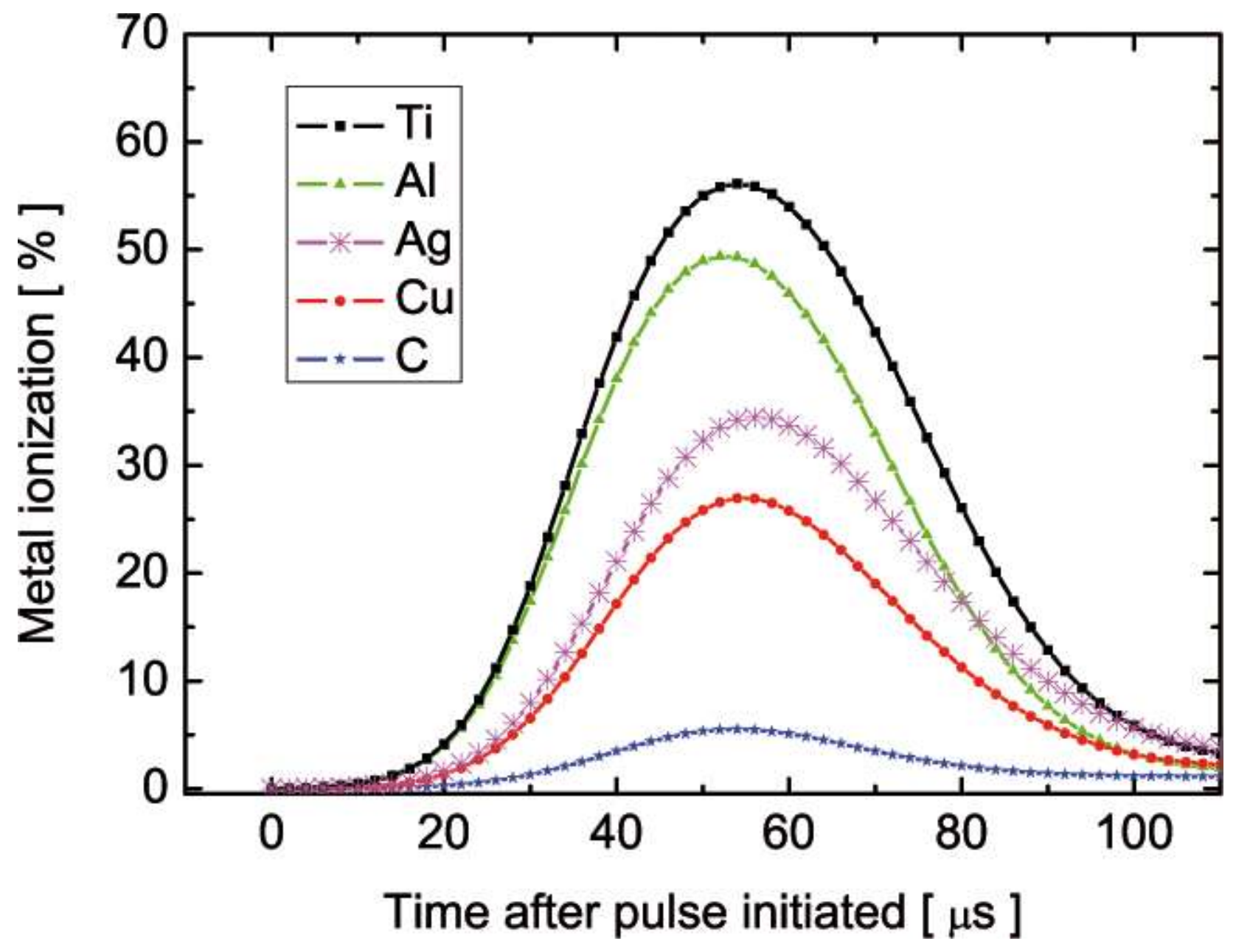

Figure 5. A comparison of the metal ionization fractions versus time from initiating the voltage pulse for different target materials. The discharge current peaks at approximately $55 \mu$ into the pulse. The results for carbon was added for comparison to highlight the difficulty to ionize some materials even at high electron density.

Figure 6 shows the calculated temporal behaviour of the electron density for the various target materials investigated in the plasma model. The electron density in the ionization region reaches values of around $10^{19}$ during the most intense part of the pulse. The reason for the similar trends in electron density independent of target material can be explained by the matching of the modelled ion current to the target and the discharge current used as input, as discussed in section 2.2. Electron densities on the order $10^{18}-10^{19} \mathrm{~m}^{-3}$ have been reported for HiPIMS discharges [36, 37]. For an electron density of the order of $10^{19} \mathrm{~m}^{-3}$ the ionization mean free path for the sputtered vapour is expected to be about $1 \mathrm{~cm}$, while for an electron density of $10^{17} \mathrm{~m}^{-3}$, common in a DCMS discharge, the ionization mean free path is approximately $50 \mathrm{~cm}$ for typical discharge conditions [38]. Thus, given the high electron density in the HiPIMS discharge we would expect a significant fraction of the sputtered metal 
to be ionized, which is also verified when looking at the densities and ionization mean free path lengths calculated using the model parameters presented in Table 1. On the contrary, in a DCMS discharge a very low fraction of the sputtered metal can be expected to become ionized on its path between the target and substrate. More importantly, the reason for the greater densification of $\mathrm{Ti}, \mathrm{Al}$, and $\mathrm{Ag}$ films compared to $\mathrm{Cu}$ films as seen in Figure 3 can be explained by the larger fraction of ionized metal, leading to an increased metal ion flux to the substrate. Here, Ti shows the largest degree of metal ionization and also the greatest density increase $(\sim 15 \%)$ followed by $\mathrm{Al}$ with approximately $6 \%$ density increase and then $\mathrm{Ag}$ of about $5 \%$ density increase. $\mathrm{Cu}$ has the lowest degree of ionization of the thin films also investigated experimentally and shows no density increase. Although the error estimations prohibit the values for individual target and film materials to be taken as absolute, an overall trend is seen.

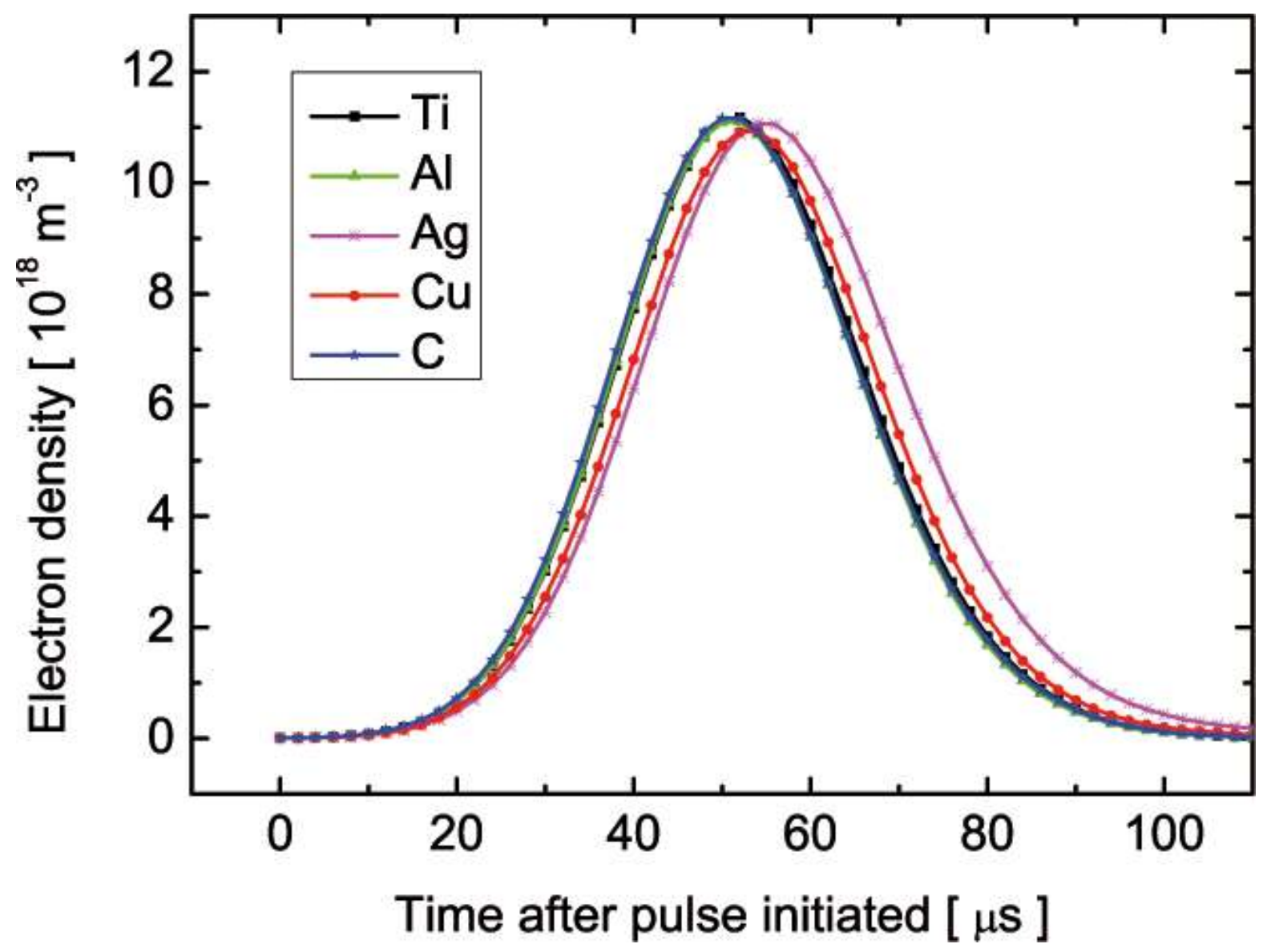

Figure 6. The electron density, $n_{e}$, versus time from initiation of the voltage pulse for the different target materials investigated. 
Note that it is the degree of ionization of the sputtered vapour that is plotted in Figure 5 , which is different from the ion flux fraction. Recall that the model simulates the ionization region and not the substrate region, meaning that the electron and ion temperature profiles necessary to compute the flux to the substrate are not determined. The ion flux is governed by the Bohm velocity, $\Gamma_{i}=0.61\left(k_{B} T_{e} / m_{i}\right)^{1 / 2} n_{i}$, and the neutral flux by the mean thermal velocity of the gas, $\Gamma_{n}=0.25\left(8 k_{B} T_{g} / \pi m_{n}\right)^{1 / 2} n_{n}$ [39]. For weakly ionized discharges the electron temperature is often significantly larger than the neutral gas temperature. This means that the ion flux fraction is larger than the fraction of ionized metal. For example, if the metal ionization is around $56 \%$ for $\mathrm{Ti}$ as seen in Figure 5 at the peak of the current, the ion flux ratio is $95 \%$ assuming that the electron temperature is $2 \mathrm{eV}$ and the neutral gas is at room temperature. This is in line with what has previously been reported by Hopwood [40]. Similar increase in the ionized flux fraction is seen for the other target materials as well. Worth mentioning is that the amount of ions as well as the electron temperature decreases rapidly after the pulse is off, which means that the neutral flux will eventually dominate the afterglow regime. It is therefore likely that the total ion flux fraction will be close to the degree of ionization for a full pulse cycle.

Finally, it should be mentioned that the model does not give any information on the energy of the ions arriving at the substrate, which means that possible densification due to higher ion energies cannot be accounted for in this study. This effect has to be studied by a molecular dynamics model or a Monte Carlo simulation.

\section{Conclusions}

In this work deposition rates and thin film density for films deposited from eight different metallic target materials have been investigated using DCMS and HiPIMS. It was found that the deposition rate for the HiPIMS process is lower than for DCMS. However, the relative deposition rates, rate $\mathrm{HiPIMS}_{\text {rate }}$ DCMS of $70 \%-85 \%$, that is observed for Ta, Pt, and $\mathrm{Ag}$ is not 
as low as have been observed in other earlier studies. The results highlights the necessity to tailor the deposition conditions with regards to source material, and that further investigations on how e.g. magnetic field configurations and pulse discharge characteristics influence film properties can be fruitful.

When comparing the two ensembles of coatings of films grown using HiPIMS with their DCMS grown counterparts, a statistically significant general trend of density increase for HiPIMS films was observed. For individual target materials, the thin film density increase could be correlated to the degree of ionization for a particular target material as assessed by a global plasma model. However, due to large uncertainties, the latter trend could not be statistically confirmed.

\section{Acknowledgements}

The authors gratefully acknowledge Daniel Magnfält for performing the profilometer measurements, and Dr. John M Noble for invaluable discussions on statistical analysis. This work was supported by the Swedish Research Council and the Swedish Foundation for Strategic Research. 


\section{References}

[1] U. Helmersson, M. Lattemann, J. Bohlmark, A.P. Ehiasarian, J.T. Gudmundsson, Thin Solid Films 513 (2006) 1.

[2] J. Bohlmark, M. Lattemann, J. T. Gudmundsson, A.P. Ehiasarian, Y. Aranda Gonzalvo, N. Brenning, U. Helmersson, Thin Solid Films 515 (2006) 1522.

[3] D. Lundin, P. Larsson, E. Wallin, M. Lattemann, N. Brenning, U. Helmersson, Plasma Sources Sci. Technol. 17 (2008) 035021.

[4] J. Alami, P.O.Å. Persson, D. Music, J.T. Gudmundsson, J. Bohlmark, U. Helmersson, J. Vac. Sci. Technol. A 23 (2005) 278.

[5] B. M. DeKoven, P. R. Ward, and R. E. Weiss, D. J. Christie, R. A. Scholl, W. D. Sproul, F. Tomasel, A. Anders, Proceedings of the 46th Annual Technical Conference Proceedings of the Society of Vacuum Coaters, May 3-8, 2003, San Francisco, CA, USA, vol., p.158.

[6] S. Konstantinidis, A. Hemberg, J. P. Dauchot, M. Hecq, J. Vac. Sci. Technol. B 25 (2007) L19.

[7] J. Alami, K. Sarakinos, F. Uslu, M. Wuttig, J. Phys. D 42 (2009) 015304.

[8] I. Petrov, F. Adibi, J. E. Greene, L. Hultman, J.-E. Sundgren, Appl. Phys. Lett. 63 (1993) 36.

[9] X. W. Zhou, H. N. G. Wadley, J. Appl. Phys. 87 (2000) 2273.

[10] F. Eriksson, N. Ghafoor, F. Schäfers, E. M. Gullikson, J. Birch, Thin Solid Films 500 (2006) 84.

[11] J. Bohlmark, J. Alami, C. Christou, A.P. Ehiasarian, U. Helmersson, J. Vac. Sci. Technol. A 23 (2005) 18.

[12] V. Kouznetsov, K. Macák, J.M. Schneider, U. Helmersson, I. Petrov, Surf. Coat. Technol. 122 (1999) 290. 
[13] J. Vlcek, P. Kudlacek, K. Burcalova, J. Musil, Europhys. Lett. 77 (2007) 45002.

[14] S. M. Rossnagel and J. Hopwood, Appl. Phys. Lett. 63 (1993) 3285.

[15] D. J. Christie, J. Vac. Sci. Technol. A 23 (2005) 330.

[16] S. Konstantinidis, J. P. Dauchot, M. Ganciu, M. Hecq, J. Appl. Phys. 99 (2006) 013307.

[17] J. Vlcek, P. Kudlacek, K. Burcalova, J. Musil, J. Vac. Sci. Technol. A 25 (2007) 42.

[18] J. Bohlmark, M. Östbye, M. Lattemann, H. Ljungcrantz, T. Rosell, U. Helmersson, Thin Solid Films 515 (2006) 1928.

[19] S. Konstantinidis, J. P. Dauchot, M. Ganciu, M. Hecq, J. Appl. Phys. 99 (2006) 013307.

[20] D. Lundin, N. Brenning, D. Jädernäs, P. Larsson, E. Wallin, M. Lattemann, M. A. Raadu,

U. Helmersson, Plasma Sources Sci. Technol. 18 (2009) 045008.

[21] U. Helmersson, M. Lattemann, J. Alami, J. Bohlmark, A.P. Ehiasarian, J.T.

Gudmundsson, Proceedings of the 48th Annual Technical Conference of the Society of Vacuum Coaters, April 23-28, 2005, Denver, CO, USA, , vol., p.458.

[22] W. Chu, J.W. Mayer, M.A. Nicolet, Backscattering spectrometry, Academic Press, New York, 1978, p 37.

[23] C. Wang, P. Brault, T. Sauvage, Eur. Phys. J. Appl. Phys. 31 (2005) 17.

[24] M. Mayer, Nucl. Instr. Meth. B, 194 (2002) 177. http://www.simnra.com/.

[25] J. R. Tesmer, M. Nastasi, Handbook of modern ion beam materials analysis, Materials Research Society, Pittsburgh, 1995, pp 127-135.

[26] S. Ashida, C. Lee, M. A. Lieberman, J. Vac. Sci. Technol. A 13(5) (1995) 2498.

[27] J. A. Hopwood, in: J. A. Hopwood (ed), Thin Films: Ionized Physical Vapor Deposition, Academic Press, San Diego, 2000, p. 181.

[28] J. T. Gudmundsson, J. Phys.: Conf. Ser. 100 (2008) 082013.

[29] Table 1 in Lee M-H, Chung C.-W. (2005) Phys Plasmas 12, 073501.

[30] Table 3 in Lee C, Lieberman MA (1995) J.Vac.Sci.Technol. A 13(2) 368-380. 
[31] R. S. Freund, R. C. Wetzel, R. J. Shul, T. R. Hayes, Phys. Rev. A 41 (1990) 3575.

[32] N. Britun, J. G. Han, and S.-G. Oh, Appl. Phys. Lett. 92 (2008) 141503.

[33] http://www.srim.org/, accessed 19 December 2008.

[34] Handbook of Chemistry and Physics $90^{\text {th }}$ edition 2009-2010 (online version), section 12 , http://www.hbcpnetbase.com/, accessed December 2009.

[35] J.L. Devore, Probability and Statistics for Engineering and the Sciences, sixth ed., Brooks/Cole, Thomson Learning Center, Belmont, CA, 2004. Chapter 9

[36] J. T. Gudmundsson, P. Sigurjonsson, P. Larsson, D. Lundin, U. Helmersson, J. Appl. Phys. 105 (2009) 123302.

[37] J. Bohlmark, J. T. Gudmundsson, J. Alami, M. Lattemann, U. Helmersson, IEEE Trans. Plasma Sci. 33 (2005) 346.

[38] J. T. Gudmundsson, Vacuum 2010, in press.

[39] M. A. Lieberman, A. J. Lichtenberg, Principles of Plasma Discharges and Materials Processing, John Wiley \& Sons, New York, 2005, p. 172.

[40] J. A. Hopwood, in: J. A. Hopwood (ed), Thin Films: Ionized Physical Vapor Deposition, Academic Press, San Diego, 2000, p 191 


\section{Tables}

Table 1. Typical plasma parameters for HiPIMS discharges. The rate coefficients $\left(k_{m i z}\right)$ for electron impact ionization of the metal atoms used in the calculations are here listed together with the references from which they were extracted.

\begin{tabular}{|c|c|c|c|c|c|}
\hline Material & $\begin{array}{c}v_{s} \\
{\left[m s^{-1}\right]}\end{array}$ & $\begin{array}{c}T_{e} \\
{[e V]}\end{array}$ & $k_{m i z}\left[m^{-3}\right]$ & $\lambda_{\text {miz }}[\mathrm{cm}]$ & $\begin{array}{l}\text { Ref. } \\
k_{\text {miz }}\end{array}$ \\
\hline Ag & 500 & 3.0 & $4.097 \times 10^{-14} T_{e}^{0.5439} e^{-6.68 / T_{e}}$ & 0.57 & 31 \\
\hline Al & 500 & 3.4 & $1.3467 \times 10^{-13} T_{e}^{0.3576} e^{-6.7829 / T_{e}}$ & 0.16 & 31 \\
\hline $\mathbf{C}$ & 500 & 3.6 & $0.4 \times 10^{-13} e^{-12.6 / T_{e}}$ & 3.8 & 27 \\
\hline $\mathbf{C u}$ & 300 & 3.2 & $3.8980 \times 10^{-14} T_{e}^{0.4840} e^{-7.1344 / T_{e}}$ & 0.37 & 31 \\
\hline $\mathbf{C u}$ & 500 & 3.2 & $3.8980 \times 10^{-14} T_{e}^{0.4840} e^{-7.1344 / T_{e}}$ & 0.62 & 31 \\
\hline $\mathbf{T i}$ & 500 & 3.4 & $2.34 \times 10^{-13} e^{-7.25 / T_{e}}$ & 0.16 & 27 \\
\hline $\mathbf{T i}$ & 700 & 3.4 & $2.34 \times 10^{-13} e^{-7.25 / T_{e}}$ & 0.23 & 27 \\
\hline
\end{tabular}

